\title{
Zystische Raumforderungen im Pankreas
}

\author{
Helmut Friess $^{a}$ Hans-Jürgen Brambs ${ }^{b}$ \\ ${ }^{\text {a }}$ Chirurgische Klinik und Poliklinik, Klinikum rechts der Isar, Technische Universität München, \\ ${ }^{\mathrm{b}}$ Klinik für Diagnostische und Interventionelle Radiologie, Universitätsklinik UIm, Deutschland
}

Zystische Raumforderungen im Pankreas werden durch die Verbesserung und Ausweitung der bildgebenden Diagnostik häufiger entdeckt. Es gibt mittlerweile eine Reihe von Berichten, die in mehrere tausend Patienten einschließenden Fallserien die Inzidenz von zystischen Pankreastumoren in einer Größenordnung von 2-3\% angeben. Die zentrale Frage bleibt: Was sollte man mit einem Patienten und dem Zufallsbefund zystischer Pankreastumor weiter tun? Symptomatische zystische Pankreastumoren bedürfen einer Therapie, und bei asymptomatischen zystischen Pankreastumoren ist eine weitergehende Diagnostik häufig notwendig, um die Dignität bzw. das potenzielle Malignitätsrisiko besser definieren zu können.

Wenngleich histopathologisch über 20 Subtypen von zystischen Tumoren im Pankreas beschrieben worden sind, so können 90\% dieser Tumoren drei Tumorentitäten zugeordnet werden:

1. Seröse Zystadenome.

2. Muzinöse Zystadenome.

3. Intraduktal papilläre muzinöse Neoplasien (IPMN).

Die Differenzierung dieser drei Tumortypen ist die Herausforderung an den Diagnostiker. Seröse Tumoren wie auch Pankreaspseudozysten sind harmlos, da sie praktisch kein Malignitätspotenzial besitzen und nur im Falle von Symptomen weiter therapiert werden sollten. Anders sieht es bei muzinösen Zystadenomen aus. Hier ist ein signifikantes Malignitätspotenzial vorhanden, und wenn keine schwerwiegenden Kontraindikationen vorhanden sind, sollten diese Prozesse reseziert werden. Bei den IPMN wurde in den vergangenen Jahren der größte Wissenszuwachs erzielt. Man unterscheidet prinzipiell zwischen Hauptgang- und Seitengang-IPMN, wobei gemischte Typen auch nicht selten vorhanden sind. Da IPMN mit Hauptgangbeteiligung ein deutlich höheres Risiko für eine maligne Entartung aufweisen als Seitengang-IPMN, orientieren sich die Therapieempfehlungen im Wesentlichen an der Wahrscheinlichkeit für eine maligne Entartung. Das Patientenalter und die Komorbiditäten sind wichtige Gesichtspunkte, die in die weitere Entscheidungsplanung mit einbezogen werden. Hauptgang-IPMN sollten reseziert werden; bei Seitengang-IPMN wird derzeit entsprechend der SendaiKonsensus-Konferenz ein differenziertes Vorgehen empfohlen. Die Zystengröße und morphologische Aspekte spielen hierbei eine gewichtige Rolle, sodass der CT/MRT-Bildgebung und der Endosonographie mit bzw. ohne Feinnadelpunktion eine hohe Bedeutung in der Therapieentscheidung zufällt. Die sich daraus ableitende Konsequenz kann von der Resektion über engmaschige Nachkontrollen bis zu großzügigen Nachkontrollen oder keinen weiteren Nachkontrollen reichen. Insbesondere die Entscheidung für die beiden Extreme fällt in der Praxis oft schwer.

Mit dem Themenheft «Zystische Raumforderungen im Pankreas» greifen wir eine aktuelle Thematik auf. Wir haben Experten gewinnen können, die sich in acht Beiträgen zu wichtigen Aspekten dieses Themenkomplexes äußern. Der Wissensgewinn aus den Erfahrungen der klinischen Praxis hat in den vergangenen Jahren insbesondere bei zystischen Pankreastumoren beträchtlich zugenommen. Die Therapiealgorithmen unterliegen fortlaufend einer kritischen Überprüfung und einer oft kontroversen Diskussion. Wir hoffen sehr, Ihnen mit diesem Themenheft eine Hilfestellung für den klinischen Umgang mit zystischen Raumforderungen im Pankreas geben zu können.

\section{KARGER \\ Fax +497614520714 \\ Information@Karger.de}

www.karger.com
(C) 2011 S. Karger GmbH, Freiburs

1662-6664/11/0273-0180\$38.00/0

Accessible online at:

www.karger.com/vim
Prof. Dr. med. Helmut Friess

Chirurgische Klinik und Poliklinik

Klinikum rechts der Isar, Technische Universität München

Ismaninger Straße 22, 81675 München, Deutschland

Tel. +41 89 4140-2121, Fax -4870

friess@chir.med.tu-muenchen.de 